Methods Human coronary artery endothelial cells (HCAEC) isolated from three different donors were cultured and stimulated with pro-inflammatory cytokines TNF- $\alpha$ and IL-1 $\beta$ $(10 \mathrm{ng} / \mathrm{ml}$ each, for 48 hours), followed by incubation with native human AcLDL cholesterol at multiple concentrations $(10-200 \mathrm{ug} / \mathrm{ml})$ for 6 hours. Cell lysates and culture supernatants were collected and analysed for IL-1 $\beta$ using ELISA. Cell viability was also measured.

Results AcLDL induced the release of IL- $1 \beta$ from stimulated HCAECs in a dose dependent manner with maximum release $(155.4 \mathrm{pg} / \mathrm{ml}, \mathrm{n}=3)$ at concentration of $50 \mathrm{ug} / \mathrm{ml}$. This was 4 fold greater than that released by cytokine-stimulated (38.49 $\mathrm{pg} / \mathrm{ml}, \mathrm{n}=3$ ) and 3 fold greater than that released by neutrophil elastase $(49.45 \mathrm{pg} / \mathrm{ml}, \mathrm{n}=3)$. This release was not caused by toxicity of the AcLDL: cell viability was confirmed by lactate dehydrogenase cell viability assay.

Conclusion AcLDL is capable of eliciting IL-1 $\beta$ release in activated HCAECs, without causing toxicity.

\section{ACCUMULATION OF CARDIOMYOCYTE SENESCENCE FOLLOWING ISCHAEMIA-REPERFUSION INJURY (IRI); A POTENTIAL THERAPEUTIC TARGET?}

${ }^{1}$ Emily Dookun*, ${ }^{2}$ Anna Walaszczyk, ${ }^{2}$ Rachael Redgrave, ${ }^{2}$ Simon Tual-Chalot, ${ }^{2}$ Oliver Yausep, ${ }^{2}$ Ioakim Spyridopoulos, ${ }^{2}$ Andrew Owens, ${ }^{2}$ Helen Arthur, ${ }^{3}$ Joao Passos, ${ }^{2}$ Gavin Richardson. 'Institute of Genetic Medicine, Newcastle University, Center for Life, Central Parkway, Newcastle Upon Tyne, UK; ${ }^{2}$ Institute of Genetic Medicine, Newcastle University; ${ }^{3}$ Institute for Ageing, Newcastle University

\subsection{6/heartjnl-2018-BCS.138}

Introduction Myocardial infarction (MI) is a prominent cause of morbidity and mortality globally. While reperfusion via primary percutaneous coronary intervention is the gold-standard therapy, it can lead to the phenomenon ischemia-reperfusion injury (IRI), characterised by progressive remodelling and heart failure. Little is understood regarding to the mechanisms driving IRI, however, increased oxidative stress is known to play a role. Our previous studies demonstrate that during ageing increased oxidative stress drives telomere associated DNA damage foci (TAF) induced cardiomyocyte senescence, which is directly associated with a hypertrophic phenotype. Furthermore senescent cardiomyocytes express a pro-fibrotic profile; in particular an up-regulation of TGF- $\beta$ expression. We now hypothesise that cardiomyocyte senescence contributes to remodelling subsequent to MI and IRI via similar mechanisms, and as such represents a potential therapeutic target.

Methods Young three month old mice underwent 60 min surgical ligation of the left anterior descending coronary artery to mimic MI followed by reperfusion. Histological analysis at numerous time points post-MI for several senescence markers was performed.

Results and conclusions In support of our hypothesis, we have observed that within the surviving myocardium, proximal to the infarct region, cardiomyocytes acquire a senescent-like phenotype. This is demonstrated by an increase in senescence markers including SA- $\beta-G a l$, p 21 and p16, in addition to an accumulation of TAF. As well as this we verified that mice displayed classical pathophysiological aspects related to MI. Using in vitro studies and transgenic mouse models we aim to better understand the biology underlying cardiomyocyte senescence, and to establish the mechanisms underlying senescence contribution to remodelling. Furthermore we aim to ascertain if modulation or clearance of cardiomyocyte senescence promotes regeneration and improves outcome following IRI.

\section{IDENTIFICATION OF HUMAN THROMBUS FORMATION IN A TRANSLATIONAL MODEL OF DEEP ARTERIAL INJURY}

Jack Andrews*, Simon Wilson, Christophe Lucatelli, Tashfeen Walton, Gillian MacNaught, Carlos Corral Alcaide, Adriana Tavares, Marc Dweck, David Newby. University of Edinburgh, SU 305 Chancellors building, 51 Little France avenue, Edinburgh, UK

\subsection{6/heartjnl-2018-BCS.139}

Background Identification of in vivo thrombus formation has the potential to assist in the diagnosis and management of acute coronary atherothrombosis. ENC2015 is a 13-amino acid fluorescent peptide analogue of 2-antiplasmin which binds to newly formed thrombus. Our aim was to assess the binding of both optical (Cy5) and PET (18F) labelled ENC2015 acute human thrombus formation in a translational model of deep arterial injury.

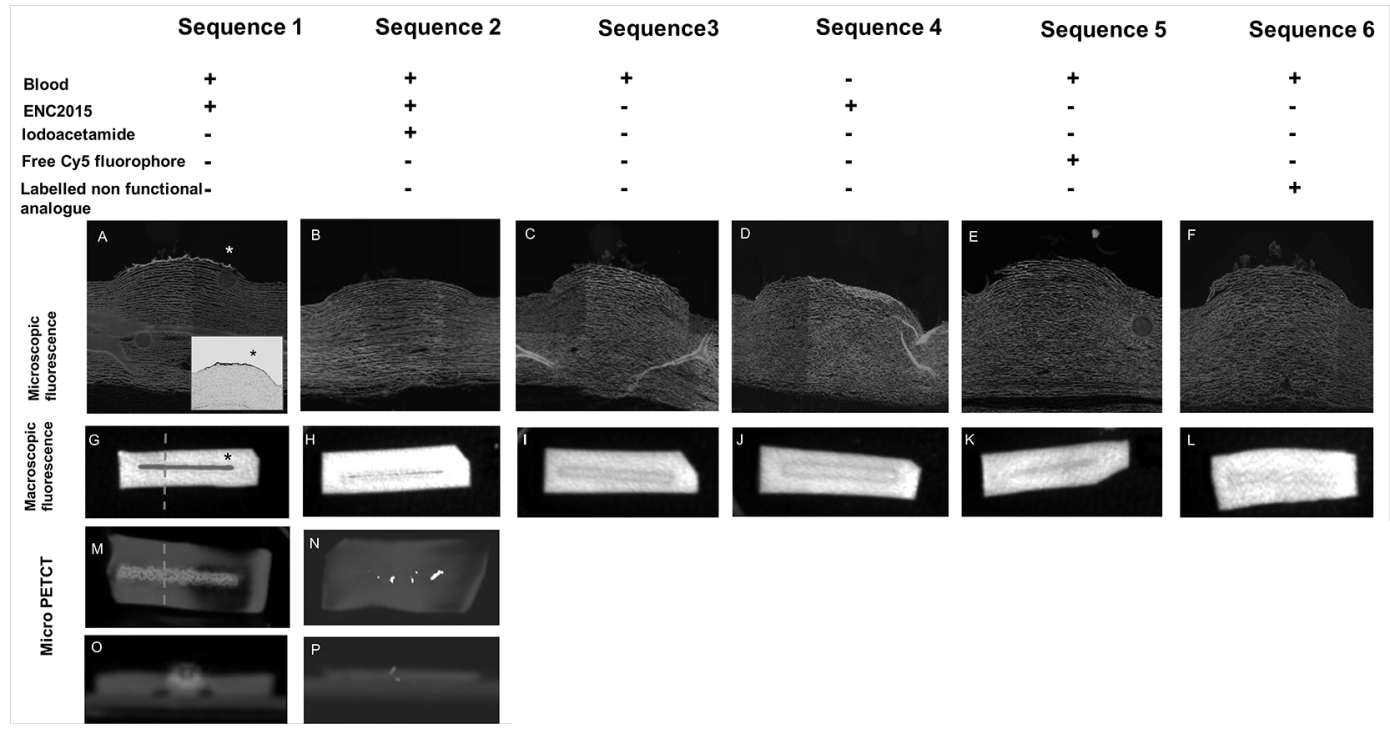

Abstract 143 Figure 1 